\title{
La escritura como práctica situada en el primer ciclo: promoción de procesos cognitivos y metacognitivos*
}

\author{
OLGA YAMIRA PEÑA GALEANO \\ molguis2208@gmail.com \\ AURA CATALINA QUINTERO SAAVEDRA ${ }^{* * *}$ \\ auracatalinaq@gmail.com
}

Recepción: 14 de octubre de 2015

Aprobación: 16 de noviembre de 2015

Forma de citar este artículo: Peña Galeano, 0.Y., \& Quintero Saavedra, A.C. (2016). La escritura como práctica situada en el primer ciclo: promoción de procesos cognitivos y metacognitivos. Cuadernos de Lingüística Hispánica, (28), 189-206. doi: http://dx.doi. org/10.19053/0121053X.4915

* Artículo de investigación. Muestra resultados de la investigación "El Modelo Metasociocognitivo. Una alternativa para situar la escritura en contexto" realizada en el marco de la Maestría en Pedagogía en Lengua Materna de la Universidad Distrital "Francisco José de Caldas", Colombia.

** Licenciada en Educación con énfasis en Educación Especial de la Universidad Pedagógica Nacional y actual maestrante en Pedagogía de la Lengua Materna de la Universidad Distrital "Francisco José de Caldas", Colombia.

** Licenciada en Educación Infantil de la Universidad Pedagógica Nacional. Especialista en Pedagogía Grupal de la Fundación Universitaria Monserrate y actual maestrante en Pedagogía de la Lengua Materna de la Universidad Distrital "Francisco José de Caldas", Colombia. 


\section{Resumen}

Este trabajo tiene como objetivo presentar resultados de la investigación titulada "El modelo metasociocognitivo. Una alternativa para situar la escritura en contexto" que está fundamentada en la perspectiva sociocultural, el modelo metasociocognitivo y la noción de cognición situada. A nivel metodológico, se aborda el diseño de Investigación Acción, por lo cual se acude a la observación participante, encuestas, entrevistas y registros de clase. En este sentido, se parte del análisis del rastreo de trabajos investigativos, acorde con tres categorías: la escritura en el ciclo uno, los procesos cognitivos y los procesos metacognitivos. Luego se presentan los resultados, organizados en seis hechos, que se derivan del análisis de los procesos de enseñanza y aprendizaje en la producción escrita de los niños en el primer ciclo: estudiantes de grado primero y segundo de básica primaria. Finalmente, se exponen las conclusiones en el ámbito pedagógico, disciplinar e investigativo.

Palabras clave:Cognición situada, composición escrita, modelo metasociocognitivo, perspectiva sociocultural, procesos cognitivos y procesos metacognitivos.

\section{Writing as a practice applied in the First and Second Grades: promoting cognitive and metacognitive processes}

\section{Abstract}

The objective of this work is to present the results obtained from the research project entitled: "The meta-sociocognitive model. An alternative for applying the writing process in a given context". This study is based on the socio-cultural perspective, the metasociocognitive model, and the notion of situated cognition. At a methodological level, this is an action-based research project that uses the following data collection instruments: participant observation, surveys, interviews and class records. In this sense, we start by the tracking and analysis of related research, according to three categories: Writing in the First and Second grades of Elementary School, cognitive processes, and metacognitive ones. After this, the results are presented and organized according to six facts derived from the analysis of the teaching and learning processes related to the written production of Elementary School students from the First and Second grades. Finally, the pedagogical, disciplinary and investigative conclusions are presented.

Key words: situated cognition, written composition, meta-sociocognitive model, socio-cultural perspective, cognitive and metacognitive processes 


\section{L'écriture en tant que pratique située au cycle élémentaire: promotion de processus cognitifs et métacognitifs}

\section{Résumé}

L'objectif de ce travail est celui de présenter des résultats de la recherche intitulée "Le modèle méta-sociocognitif. Une alternative pour situer l'écriture en contexte" dont le fondement est la perspective socioculturel, le modèle méta-sociocognitif et la notion de cognition située. Au niveau méthodologique, on aborde le dessin de Recherche Action, ce qui fait que l'on recoure à l'observation participante, des enquêtes, des interviews et des enregistrements de cours. Dans ce sens, on part de l'analyse du traînement de travaux de recherche, selon trois catégories: l'écriture dans le cycle élémentaire, les processus cognitifs et les processus métacognitifs. Ensuite, on présente les résultats, organisés en six faits, qui se dérivent de l'analyse des processus d'enseignement et d'apprentissage dans la production écrite des enfants dans le cycle de base: des élèves de CP et de CE1. Finalement, on expose les conclusions aux domaines pédagogique, disciplinaire et de recherche.

Mots clés: Cognition située, composition écrite, modèle méta-sociocognitif, perspective socioculturelle, processus cognitifs et processus métacognitifs.

\section{A escritura como prática situada no primeiro ciclo: promoção de processos cognitivos e metacognitivos}

\section{Resumo}

Este trabalho tem como objetivo apresentar resultados da pesquisa intitulada " 0 modelo metasociocognitivo. Uma alternativa para situar a escritura em contexto" que está fundamentada na perspectiva sociocultural, no modelo metasociocognitivo e na noção de cognição situada. No nível metodológico se aborda o desenho de Pesquisa Ação, pelo qual se recorre à observação participante, inquéritos por sondagem, entrevistas e registros de aula. Neste sentido, parte-se da análise do rastreamento de trabalhos de pesquisa, de acordo com três categorias: a escritura no ciclo um, os processos cognitivos e os processos metacognitivos. Depois se apresentam os resultados, organizados em seis fatos, que se derivam da análise dos processos de ensino e de aprendizagem na produção escrita das crianças no primeiro ciclo: estudantes de primeiro e segundo ano de primária. Finalmente, se expõem as conclusões no âmbito pedagógico, disciplinar e de pesquisa.

Palavras chave: Cognição situada, composição escrita, modelo metasociocognitivo, perspectiva sociocultural, processos cognitivos e processos metacognitivos. 


\section{Introducción}

Las investigaciones acerca de la lengua materna y específicamente de la modalidad escrita se han transformado y evolucionado de acuerdo con la perspectiva desde la cual se han abordado, a partir de los postulados de Ferreiro (1982), se pueden reconocer corrientes como la lingüística, la psicolingüística y la sociocultural. La primera perspectiva está centrada específicamente en aspectos relacionados con el texto, la segunda considera la escritura como un proceso cognitivo y la tercera involucra el contexto como aspecto fundamental de la composición escrita reconocida por Cassany (1999) como una práctica social y cultural.

En la línea sociocultural se inscribe el modelo metasociocognitivo propuesto por Arroyo (2009) que se constituye en una propuesta integradora de la composición escrita al involucrar procesos cognitivos, metacognitivos y socioculturales, el cual reconoce la "escritura como proceso" articulada a la noción de cognición situada de Rogoff (1997), quien concibe la composición escrita en un marco social, cultural y discursivo determinado.

Desde esta perspectiva, se realiza el proyecto de investigación "El modelo metasociocognitivo. Una alternativa para situar la escritura en contexto" que se desarrolla en la Maestría de Pedagogía en Lengua Materna sustentado en el modelo de composición escrita metasociocognitivo, el cual se circunscribe al enfoque sociocultural basado en los postulados de Vygotsky (1931). De esta manera, se constituye en el objetivo central del presente estudio determinar el papel que desempeñan los procesos cognitivos y metacognitivos propuestos en el Modelo Metasociocognitivo para el desarrollo de los procesos de producción escrita de los niños en el ciclo uno.

En primer lugar, se hace referencia a los antecedentes investigativos que dan cuenta del estado actual de la escritura en el ciclo uno como campo de estudio. En segundo lugar, se explica el modelo metasociocognitivo como la perspectiva teórica de escritura que sustenta el proceso investigativo. En tercer lugar, se exponen los referentes metodológicos fundamentados en el paradigma cualitativo y el enfoque de investigación acción que orienta las acciones propias de la actividad investigativa. En cuarto lugar, se presentan resultados a manera de los hallazgos encontrados y en diálogo con la perspectiva sociocultural, el modelo metasociocognitivo y la noción de cognición situada. Para finalizar con las conclusiones del proyecto en el ámbito pedagógico, disciplinar e investigativo. 


\section{Antecedentes de la investigación: escritura y metacognición}

Con la finalidad de identificar el estado actual del tema por investigar, novedades, alcances y límites de los estudios efectuados hasta el momento, se realiza el rastreo y análisis de 25 trabajos investigativos en Dialnet, Proquest y Redalyc desarrollados a nivel de maestría y doctorado en Colombia, Argentina, Venezuela, México y España, entre el año 2010 hasta el 2015, a partir de tres categorías: la escritura en el ciclo uno, los procesos cognitivos y metacognitivos.

De esta manera, se lleva a cabo un análisis documental de tipo descriptivo e interpretativo, referido a la identificación de los objetivos investigativos y al énfasis dado al objeto de estudio. Como resultado se identifican seis subcategorías que dan cuenta de los enfoques que han tenido las investigaciones desarrolladas en torno a la escritura en el primer ciclo:

1. Concepciones teóricas y metodológicas de los docentes: se encuentran cinco estudios que se caracterizan por centrar su interés en el pensamiento de los maestros y las prácticas pedagógicas relacionadas con la enseñanza de la escritura.

2. Desarrollo de elementos formales de la escritura: se hallan tres propuestas orientadas a la comprensión de las etapas de la escritura en lo referente a aspectos particulares del texto escrito.

3. Descripción del proceso escritor: se encuentran tres investigaciones que se caracterizan por dar cuenta de los procedimientos lingüísticos realizados por los niños en la producción escrita de textos.

4. Evaluación del impacto de propuestas pedagógicas para el desarrollo del proceso de escritura: se analizan seis trabajos que evidencian la preocupación de los docentes investigadores por cualificar los procesos escriturales de los estudiantes a través del diseño, implementación y posterior evaluación del impacto de diferentes propuestas pedagógicas.

5. Relación entre escritura y lectura: se encuentran dos investigaciones basadas en el modelo interactivo e integral de la composición escrita, cuyo interés es presentar de la lengua escrita procesos referidos a la comprensión (lectura de textos) y a la producción (textos orales y escritos) de la misma.

6. Relación entre proceso escritor, cognición y metacognición: se encontraron cinco investigaciones que conciben la escritura como un proceso y al estudiante como un sujeto activo, capaz de desarrollar habilidades para reconocer, controlar y regular 
sus propios procesos cognitivos. Es importante aclarar que solo uno de los trabajos de esta categoría aborda el estudio de los procesos metacognitivos en los niveles iniciales de formación.

Si bien, son muy importantes los aportes realizados por las investigaciones con respecto a las prácticas de enseñanza y procesos cognitivos de la composición escrita en el primer ciclo, es evidente el vacío en la investigación de procesos metacognitivos en la escritura de los niños en los primeros grados de escolaridad; no obstante, la escritura como proceso metacognitivo es abordada en las investigaciones rastreadas en niveles de bachillerato y universidad. Razón por la cual es relevante y necesario profundizar y aportar conocimiento pedagógico e investigativo a este campo de estudio en los niveles iniciales de escolaridad.

\section{La escritura en contexto: Modelo metasociocognitivo y cog- nición situada}

La escritura como campo de estudio del presente proyecto de investigación es concebida como un proceso cognitivo, comunicativo y discursivo en el marco del Modelo Metasociocognitivo de Arroyo (2009), en articulación con la teoría de Vygotsky (1931) sobre la perspectiva sociocultural.

A nivel cognitivo, según Flower \& Hayes (1996), la escritura además de la apropiación del código involucra una serie de operaciones mentales que determinan la forma como se genera, se relaciona y se organiza un texto a través de subprocesos como la planificación, la textualización y la revisión. Por otra parte, Cassany (1999) aporta el reconocimiento de la escritura como proceso comunicativo en cuanto a la característica social de intencionalidad de expresión hacia otros y como proceso discursivo en el sentido con el que se produce un texto desde la participación activa e interacción que el escritor establece con otros miembros de una comunidad escritora acorde a un contexto específico.

Entendida de esta manera, la escritura como proceso cognitivo que se proyecta dentro de un contexto requiere contemplar una serie de elementos explicitados por Camps (2003) como la selección de un tipo de texto, el reconocimiento de un destinatario determinado y la identificación de una intencionalidad comunicativa en particular. Entonces, si la composición escrita está determinada por una serie de procesos cognitivos propios de cada sujeto en dinámicas de interacción hacia otros, en palabras de Flórez y Torrado et al. (2003), es importante la toma de decisiones sobre la composición escrita por parte del niño como sujeto escritor para ser consciente de su propio proceso, controlarlo y regularlo, es decir, que lleve a cabo actividades de tipo metacognitivo. 
A partir de lo expuesto anteriormente, es importante decir que el presente proyecto parte de una concepción de escritura que toma como elemento central el concepto de cognición situada desde la perspectiva sociocultural expuesta por Díaz Barriga (2003), quien considera que "el conocimiento es situado, es parte y producto de la actividad, el contexto y la cultura en que se desarrolla y utiliza" (p. 2). En esta medida, escribir es considerada una práctica social y una construcción cultural que contempla una serie de procesos cognitivos que no se dan de forma abstracta o aislada del contexto, sino que por el contrario estas operaciones mentales están ligadas a las acciones e interacciones que los sujetos llevan a cabo dentro de comunidades discursivas particulares.

De acuerdo con Díaz Barriga (2003), el conocimiento situado de la escritura exige una mirada del niño como sujeto escritor que hace parte de una comunidad específica en la cual se relaciona e interactúa con otros escritores, y cuyos escritos se derivan y adquieren sentido en las relaciones dialógicas que se establecen con el entorno social y cultural. Es importante aclarar que, desde esta perspectiva, los procesos metacognitivos de la escritura resultan necesarios para generar prácticas reales de composición escrita como lo exponen Cuervo \& Flórez (2000), con la finalidad de que los estudiantes sean escritores activos con conciencia y capacidad para regular sus propios procesos, de manera que sea posible:

hacer de la escuela una comunidad de escritores que producen sus propios textos para dar a conocer sus ideas, para informar sobre hechos que los destinatarios necesitan o deben conocer, para incitar a sus lectores a emprender acciones que consideren valiosas para convencerlos de los puntos de vista o las propuestas que intentan promover, para protestar o reclamar, para compartir con los demás una bella frase 0 un buen escrito, para intrigar o hacer reír (Lerner, 2001, p. 26).

Sin embargo se encuentra, a partir de la lectura del contexto particular, que en las instituciones educativas las acciones pedagógicas relacionadas con la producción escrita en el primer ciclo, como lo señala Fernández Cárdenas (2009), se caracterizan por ser descontextualizadas. Se trata de prácticas de carácter repetitivo y mecánico basadas en el método sintético (alfabético o silábico), las cuales dan prioridad al aprendizaje de los signos lingüísticos y reflejan la ausencia de actividades de tipo cognitivo y metacognitivo, es decir, como lo expresa Flórez \& Torrado (2003), no se promueve la conciencia, el control y la regulación de los propios procesos de producción escrita infantil.

En este sentido, se hace necesario tomar como eje el desarrollo de procesos cognitivos y metacognitivos en la producción escrita, los cuales están ligados a las acciones reales o contextos compartidos que posibilitan la reconstrucción de sentido e interpretación de textos dentro de lo que conceptualiza Camps (2003), como comunidades discursivas. 
Razón por la cual, se acude a la noción de cognición situada para hacer de la escritura una práctica contextualizada, en lo que es significativo: planear lo escrito, leerlo, dialogarlo y reescribirlo pensando en un posible lector; y de esta manera lograr que los niños sean sujetos conscientes con capacidad para controlar y regular su propio proceso escritor.

\section{Metodología}

Este trabajo se realiza en el marco de la Maestría en Pedagogía de la Lengua Materna de la Universidad "Francisco José de Caldas" y en la línea de investigación Pedagogía de las Actividades Discursivas de la Lengua. Puesto que se realiza la reflexión e investigación pedagógica, en torno a la modalidad escrita de la lengua, en la medida que el proyecto investigativo reconoce la relevancia del carácter social y cultural de la escritura como eje de los procesos de aprendizajes en el contexto escolar.

El presente proyecto parte de la concepción de Vasilachis (1997), sobre los paradigmas como "[...] marcos teóricos-metodológicos utilizados por el investigador para interpretar los fenómenos sociales en el contexto de una sociedad determinada" (p. 30), con la particularidad de orientar el proceso investigativo en el acercamiento, interpretación e intervención en la realidad educativa, en la cual se reconoce el problema de investigación. En otras palabras, se trata de comprender que el docente está directamente relacionado con el proceso investigativo, puesto que es él quien escucha, observa, explora, interpreta, construye significados y toma decisiones en el devenir de la investigación.

El presente trabajo se acoge al paradigma interpretativo porque los hechos objeto de estudio, como lo expresa Vasilachis (1992), se analizan y se interpretan en contextos reales como construcciones sociales en directa relación con las características propias de los sujetos que hacen parte de la realidad investigada, incluido el investigador. De esta manera, las docentes investigadoras, a través de la continua reflexión sobre sus propias prácticas, contribuyen a contextualizar y transformar las prácticas de enseñanza y aprendizaje de escritura en relación con los procesos cognitivos y metacognitivos.

Desde la perspectiva del paradigma interpretativo, esta investigación asume el enfoque cualitativo, ya que como lo expone Martínez (2006), "trata de identificar la naturaleza profunda de las realidades, su estructura dinámica, aquella que da razón plena de su comportamiento y manifestaciones" (p. 128). Es decir, implica comprender la escuela como espacio de reconstrucción de significados por medio de la reflexión y estructuración de acciones sistemáticas que deriven en el reconocimiento, comprensión y transformación de las acciones pedagógicas, a partir del análisis de las dinámicas que se establecen entre los elementos y actores involucrados en los procesos educativos. 
De acuerdo con Guardián Fernández (2007), a partir del enfoque cualitativo se comprenden las relaciones de saber y poder en la escuela desde la subjetividad, participación e interacción del investigador con la comunidad educativa, de modo que, los actos humanos de maestros y estudiantes cobran significado para el proceso investigativo. Motivo por el cual, en el caso de esta investigación, identidad y subjetividad de las docentes investigadoras están presentes al indagar, interpretar e intervenir sobre los procesos cognitivos y metacognitivos en la enseñanza de la composición escrita en el ciclo uno.

A su vez, la investigación opta por el diseño de Investigación Acción, puesto que aborda el plano del ser y el hacer de los sujetos inmersos en los procesos de enseñanza y aprendizaje dentro de un contexto escolar específico. En palabras de Guardián Fernández (2007), este diseño ubica la investigación en los problemas prácticos cotidianos vivenciados por los docentes investigadores, ante lo cual el docente como investigador es el más acertado en la construcción de conocimiento pedagógico en aras de la transformación de su práctica educativa.

El diseño de la Investigación Acción permite dar sentido a la presente investigación, ya que de acuerdo con Elliot (2000), analiza "las acciones humanas" como problemáticas, susceptibles de cambio y que requieren una respuesta práctica (p. 24). En consecuencia, se reconoce la importancia de desarrollar acciones que permitan la transformación de las prácticas cotidianas de los docentes, lo cual incluye la reflexión de la propia práctica pedagógica y el rol de cada participante, en el caso de la producción escrita en el ciclo.

En coherencia con el diseño de investigación, son partícipes del proceso investigativo en mención las dos docentes investigadoras con sus respectivos estudiantes, los cuales conforman un grupo de 80 niños de grado 2 do. con edades comprendidas entre los seis y siete años, quienes a través de la reflexión sobre sus acciones pedagógicas, contribuyen a contextualizar la problemática de investigación, específicamente en lo relacionado con las prácticas de enseñanza y aprendizaje de escritura realizadas en dos colegios distritales de las localidades de Engativá y Suba.

\section{Fases de la investigación}

Se asumen tres fases en el diseño de Investigación Acción, planteadas por Guardián Fernández (2007), a saber: preactiva, interactiva y posactiva, las cuales en correspondencia con la elección del método investigativo y la perspectiva teórica del investigador, le implica al docente definir un tipo de intervención en la realidad educativa (unidades didácticas, proyecto de aula o talleres), recursos y tiempo. Ahora bien, Elliott (2000) plantea que es el docente quien se encuentra directamente vinculado al contexto escolar, por lo cual su función como investigador se orienta a transformar la realidad educativa. 
La fase preactiva, para Guardián Fernández (2007), consiste en reflexionar y analizar las concepciones, además de construir fundamentos de la investigación a nivel ontológico-axiológico, epistemológico y metodológico. En este sentido, se desarrolló un proceso de codificación, categorización y triangulación de los corpus recogidos como documentos institucionales, cuadernos, guías y escritos de los niños.

A partir de la información recolectada, se da paso a lo que Elliott (2000) denomina "interpretar lo que ocurre" desde quienes actúan e interactúan en la situación problema que valida los relatos del docente investigador en los diálogos con los participantes y así, identificar una ausencia, interés o particularidad en los procesos de enseñanza y aprendizaje que sean susceptibles de ser investigados en el contexto escolar en el que el docente investigador es partícipe. Proceso que, en el caso particular de este trabajo, se basa en el análisis de registros de clase con respecto a tres ejes temáticos: concepciones, prácticas pedagógicas y desempeños de los niños.

La fase interactiva, acorde con Guardián Fernández (2007), hace referencia a la selección y desarrollo de la propuesta de intervención con base en un cronograma establecido para el proceso investigativo. Es así como en esta etapa, según Martínez (2006), se hace plausible la representatividad de los actores involucrados en la investigación, como "coinvestigadores", en la medida en que son sus voces, experiencias y acciones las que dan sentido y orientan la investigación.

Como propuesta de intervención, se opta por la formulación del proyecto de aula definido por Rincón (2012), como una estrategia didáctica de la metodología del trabajo por proyectos que contempla como componentes: proyecto de acción, global de aprendizaje y específicos de construcción de competencias. A su vez, para Jolibert y Sraike (2009), permite abordar la producción textual auténtica en lo procesual y funcional hacia la reflexión metacognitiva de los aprendizajes mediante el trabajo cooperativo y democrático de tipo socioconstructivista.

La fase posactiva, propuesta por Guardián Fernández (2007), consiste en el tratamiento de la información y divulgación de avances y resultados, lo cual es posible durante y al finalizar la investigación. Esta fase implica un "comportamiento reflexivo" de toma de decisiones justificadas y una responsabilidad directa con el contexto educativo en lo referente a los hallazgos del estudio realizado.

\section{Hallazgos de la investigación}

A continuación, se presentan los hallazgos resultantes de la primera fase de la investigación (preactiva) que involucra la recolección, la triangulación y el análisis de la 
información proveniente de tres campos: etnográfico, político y cartográfico, los cuales representan las particularidades de la realidad educativa por investigar. De esta manera, surgen tres categorías de análisis: la producción escrita, los procesos cognitivos, y los procesos metacognitivos.

En este sentido, en palabras de Elliott (2000), se reconocen como "hechos" a aquellas situaciones interpretadas por las docentes investigadoras, que dan sustento al proceso investigativo. De esta manera, a partir de la lectura etnográfica del contexto, se identifican seis grandes hechos, los tres primeros hacen referencia a las prácticas pedagógicas generales en el ciclo uno y los tres siguientes, a la reflexión de la propia práctica pedagógica de las docentes investigadoras:

Contradicción entre los fundamentos pedagógicos expuestos en los documentos políticos, educativos e institucionales y las propuestas pedagógicas desarrolladas en las prácticas de enseñanza de escritura.

1. Contradicción entre las concepciones de escritura de los docentes y sus prácticas pedagógicas.

2. Desconocimiento por parte de los docentes de los procesos metacognitivos en relación con la escritura.

3. Producciones escritas enfocadas hacia el género narrativo.

4. Desarrollo de prácticas pedagógicas de producción escrita que desconocen los procesos cognitivos y metacognitivos.

5. Las producciones escritas de los niños tienen como único destinatario o lector al docente.

A partir de la triangulación de la información proveniente de los documentos políticos e institucionales, surge el primer hecho, la contradicción entre los fundamentos pedagógicos y las propuestas pedagógicas desarrolladas en las prácticas de enseñanza y aprendizaje de la escritura. Los documentos políticos se refieren a los Estándares básicos de competencias del lenguaje (de $1^{\circ}$ a $3^{\circ}$ ), Lineamientos Curriculares y los Referentes para la Didáctica del Lenguaje en el Primer Ciclo, en cuanto a los archivos institucionales, se analizan: el PEI, Plan de área de Humanidades, Propuesta Curricular, Plan de estudios y boletines de grado de ciclo uno.

Respecto del enfoque pedagógico planteado en los documentos políticos, este se encuentra en correspondencia con la perspectiva que sustenta las prácticas de enseñanza 
de las dos instituciones educativas distritales, pues tanto en lo político como en lo institucional se retoma el constructivismo como teoría de aprendizaje que orienta las acciones pedagógicas de los procesos de enseñanza y aprendizaje de la lengua escrita como un proceso cognitivo y comunicativo. Sin embargo, no hay relación entre la postura pedagógica constructivista y los propósitos de aprendizaje de los boletines, pues están formulados en términos de adquisición y manejo del código alfabético; se apoyan en los enfoques estructuralistas y normativos de la enseñanza de la lengua escrita.

El segundo hecho, la contradicción entre las concepciones de escritura de los docentes y sus prácticas pedagógicas, se sustenta en el análisis de las encuestas realizadas a las docentes sobre la escritura, en contraste con las evidencias del trabajo pedagógico en el aula (cuadernos, guías y evaluaciones) y en las entrevistas realizadas a los niños, cuyas voces dan cuenta de las prácticas de enseñanza de sus maestras, con énfasis en la adquisición del código. Acciones pedagógicas que son motivo de reflexión por parte de la Secretaría de Educación Distrital (SED, 2006), a partir de los trabajos de Emilia Ferreiro, Liliana Tolchinski, Ana Teberosky, Gloria Rincón y Olga Villegas, en la medida en que coinciden en afirmar que el código alfabético convencional es un punto de llegada más que de partida en el desarrollo de la significación del niño; por lo tanto, el énfasis de los procesos de enseñanza y aprendizaje debe estar en la construcción del sentido social de la escritura.

Con base en lo anterior, se analizaron las concepciones de las maestras en torno a la escritura mediante una encuesta basada en las siguientes preguntas: ¿qué es escribir?, ¿qué significado tiene para usted la escritura?, ¿cómo considera que aprenden los niños a escribir?, ¿qué escriben sus estudiantes?, y ¿cuál cree que es la mejor forma de enseñar a escribir? Los resultados de estas encuestas permiten determinar que las docentes conciben la escritura como un proceso o habilidad que les posibilita a los niños expresar sus ideas y comunicarse con otros. En cuanto a la enseñanza de la escritura, las docentes refieren aspectos que se deben tener en cuenta como son los intereses de los estudiantes, la motivación y la utilización de la escritura como medio de comunicación.

A partir del análisis de las voces de los niños (entrevistas con respecto a ¿qué es escribir?, ¿cómo aprendiste a escribir?, y ¿para qué escribes?) y de las muestras de los cuadernos, guías y evaluaciones (presencia de sellos de letras, planas de combinaciones silábicas y de palabras, dictados y transcripciones de textos escolares), se pudo determinar que a pesar de lo expresado por los docentes acerca de los procesos de enseñanza y aprendizaje enmarcados dentro de perspectivas constructivistas, las acciones pedagógicas demuestran que detrás de ellas se evidencian concepciones netamente tradicionales. 
En palabras de Jaimes (2001), según la lingüística tradicional (naturalistas convencionalistas) y el pensamiento metafísico (presocráticos) "[...] la lengua es concebida como una sumatoria de elementos, un inventario de palabras [...] y la gramática como el arte de hablar y escribir correctamente" (p. 112). Por ende, no hay correspondencia entre el discurso y las prácticas, puesto que en las aulas predominan actividades que hacen énfasis en la memorización, repetición y reproducción de sílabas y palabras, siendo común la utilización de los sellos, la realización de las planas y el dictado.

El desconocimiento, por parte de los docentes, de los procesos metacognitivos en relación con la escritura se detectó en el análisis de las entrevistas realizadas a los del ciclo uno, apoyadas en tres preguntas: ¿cómo se relaciona la escritura con los procesos cognitivos de los estudiantes?, ¿qué diferencia hay entre lo cognitivo y metacognitivo?, y ¿qué estrategias metacognitivas utiliza en el aula para la enseñanza de la escritura? De esta manera, se pudo establecer a través de las respuestas dadas por los docentes la falta de claridad acerca del concepto de procesos cognitivos, los cuales se relacionan con las operaciones mentales que llevamos a cabo en el aprendizaje y la solución de problemas, tales como: la atención, la memoria, las relaciones que se establecen entre la información y la aplicación de conocimientos, entre otras. Por este vacío conceptual, hay una imposibilidad para explicar el concepto de procesos cognitivos y metacognitivos, y, por ende, para diferenciarlos.

Debido al desconocimiento que muestran las docentes entrevistadas acerca de los procesos metacognitivos, se encontró que no se promueve el uso de este tipo de estrategias en las actividades relacionadas con la producción escrita, las docentes asocian de manera errónea el concepto de estrategias metacognitivas con algunas acciones de tipo metodológico que llevan a cabo con sus estudiantes.

Con respecto al cuarto hecho, las producciones escritas de los niños centradas en textos narrativos, se encuentra que las docentes privilegian la composición textual de escritos de tipo narrativo y descriptivo, tales como: cuentos, anécdotas de situaciones de la cotidianidad, comentarios sobre programas de televisión y descripción de objetos, animales, personas y lugares. Es así como en las acciones pedagógicas encaminadas a la producción escrita en el ciclo uno, se desconoce el trabajo de otros géneros textuales diferentes al narrativo, aspecto que condiciona la expresión escrita de los estudiantes.

El reto de la escuela es desarrollar la autonomía de los niños para la producción de diferentes tipos de texto, en palabras de Jolibert y Sraïke (2009), las prácticas de escritura han de fomentar en los niños la capacidad "[...] de actuar, de reaccionar o hacer actuar a partir de todo tipo de escritos" (p. 13), lo cual solo se logra en la medida en que el 
docente propicie la lectura y la escritura de diversos géneros textuales, aspecto que además favorece el acceso a la información que circula en diferentes comunidades discursivas.

La quinta situación o hecho, el desarrollo de prácticas pedagógicas de producción escrita que desconocen los procesos cognitivos y metacognitivos, se derivó del análisis de los registros de clase que dan cuenta de las propias prácticas pedagógicas de las docentes investigadoras en relación con la producción escrita en el grado segundo de primaria. Se encuentra que aunque hay mayor claridad conceptual por parte de las docentes en cuanto al significado de procesos cognitivos y metacognitivos, y la importancia de estos frente a la producción escrita, se refleja que el discurso pedagógico difiere de las prácticas de enseñanza y los procesos de aprendizaje de la escritura que se fomentan.

Dado que los niños realizan la escritura del texto narrativo solo una vez, sin contemplar la elaboración de planes textuales y borradores, se carece de revisiones del texto y, por ende, la práctica escritural como el proceso. Así, toma validez lo planteado por Cassany (2001), quien expone "Prestar atención a las producciones intermedias (listas, esquemas, borradores, etc.) fomenta la concepción de que la escritura es mucho más que el producto final, que abarca todo el proceso de elaboración y textualización del significado." (p. 3).

Entonces, en la medida en que las docentes se centran en la escritura definitiva, niegan la posibilidad de una práctica susceptible de ser reelaborada las veces que sea necesario con respecto a pensar qué y cómo se va a escribir un texto determinado. Por consiguiente es claro, en primer lugar, la falta de desarrollo de procesos cognitivos en la escritura, tales como planificación, textualización y revisión; y en segunda instancia, la ausencia de toma de decisiones y cambios realizados en el texto durante su composición, acciones relacionadas con los procesos metacognitivos de control y autorregulación de la escritura.

En referencia al sexto hecho, las producciones escritas de los niños tienen como único destinatario o lector al docente. Es evidente que los estudiantes realizan sus producciones escritas sin responder a intenciones comunicativas reales o a actividades discursivas acordes a un contexto en particular. En consecuencia, dichos escritos tienen como único propósito cumplir con acciones académicas articuladas al desarrollo de ejes temáticos contemplados en el plan de estudios, razón por la cual, las producciones escritas de los niños tienen como único espacio de circulación el aula y al docente como único lector.

En todos los casos, será necesario que las tareas de escritura estén encuadradas en una situación comunicativa concreta y tengan un propósito determinado, a fin de 
que el alumno sea consciente de la pertinencia e importancia de emitir su mensaje en un contexto sociocultural específico. Ello puede darse, con mayor eficacia, si encuadramos la enseñanza de la escritura en un proyecto interdisciplinario (Lacon \& Ortega, 2008, p. 250).

Al comprender la producción escrita como una práctica sociocultural situada que involucra procesos cognitivos y metacognitivos, esta adquiere significado y sentido dentro de comunidades discursivas reales como lo expone Rogoff (1997), quien plantea que se deben tener en cuenta tres planos en el análisis de la actividad considerada sociocultural: apropiación participativa, participación guiada y aprendizaje, estos tres planos se relacionan con procesos personales, interpersonales y comunitarios. En palabras de Díaz Barriga (2003): “[...] en la perspectiva de la cognición situada, el aprendizaje se entiende como los cambios en las formas de comprensión y participación de los sujetos en una actividad conjunta" (p. 5), entonces, se contempla además de la interacción entre el sujeto y el contexto, las dinámicas de relación que surgen a partir de actividades en las cuales los niños, como escritores auténticos, se proyectan a otras comunidades discursivas fuera del aula mediante experiencias, tales como: participación en convocatorias de publicación en el periódico escolar o en redes de textos infantiles y socialización de producciones textuales a la comunidad educativa.

Finalmente, de acuerdo con las seis situaciones o hechos mencionados, resulta relevante la presente investigación para fortalecer la producción escrita de los estudiantes como práctica situada, a través de la articulación de los procesos cognitivos y metacognitivos en conjunto con los socioculturales. Para lo cual, en correspondencia con Fons (2004), implica generar prácticas de enseñanza y aprendizaje de la escritura, basadas en situaciones reales de uso de la lengua, orientadas a la regulación consciente de los procesos de composición escrita. Se trata de llevar a cabo los procesos cognitivos (planeación, textualización, revisión y edición) y metacognitivos mediante el desarrollo de la evaluación a lo largo del proceso de producción escrita, a partir de herramientas como las tablas de especificaciones y las listas de cotejo según las características de la tipología textual por trabajar, las cuales son elaboradas en conjunto con los niños, con la finalidad de que puedan monitorear y controlar su propio proceso escritor; ello les permitirá tomar conciencia de él y cualificarlo. 


\section{Conclusiones}

Con la finalidad de transformar las prácticas de enseñanza de la escritura basadas en la repetición, reproducción y memorización de unidades lingüísticas aisladas, como lo expone Ferreiro (1982), se hace necesario comprender que escribir a nivel cognitivo va más allá de la apropiación del código, dado que involucra una serie de operaciones mentales que determinan la forma como se genera, se relaciona y se organiza un texto, a través de subprocesos como la planificación, la textualización y la revisión. Ahora bien, como proceso discursivo requiere pensar en el sentido con el que se produce un texto desde la participación activa e interacción que el escritor establece con otros miembros de una comunidad, es decir, se trata de comprender la composición escrita en su uso funcional como una práctica con sentido y en contexto.

En lo referente a los procesos de enseñanza y aprendizaje de la composición escrita desde la perspectiva sociocultural y el modelo metasociocognitivo, es importante reconocer el carácter situado del proceso escritor, el cual se complejiza y se enriquece junto con otros, entre pares y maestros, quienes acompañan el proceso escritor. Se trata de pensar y propiciar la escritura como un acto individual y a la vez colectivo, que adquiere sentido en las acciones reales y cotidianas de los sujetos escritores en función de sus interacciones y dinámicas comunicativas con los miembros de su comunidad discursiva.

Desde el carácter disciplinar de la lengua escrita, el modelo metasociocognitivo plantea los procesos cognitivos y metacognitivos como factores esenciales para hacer partícipes a los niños en una comunidad escrita de construcción de significados, regulación de su proceso a partir de la resolución de problemas y toma de decisiones al componer un escrito.

En cuanto al punto de vista pedagógico, la perspectiva sociocultural aporta a la transformación de las prácticas de enseñanza de la escritura en la medida que los niños se apropien de su proceso escritor y aprendan de este. Lo cual le implica al docente brindar espacios de escritura auténtica para lograr que los niños sean sujetos activos de la comunidad escrita a la cual pertenecen y conscientes de su propio proceso escritor.

Con respecto al ámbito metodológico en la perspectiva del diseño de investigación acción, tras identificar como problemática investigativa en el contexto educativo la ausencia del uso de estrategias metacognitivas en el proceso escritor, se hace necesario intervenir como docentes investigadores de la acción educativa, a través de la interpretación, comprensión y participación en el contexto educativo desde el trabajo por proyectos de aula; dado que como propuesta de intervención pedagógica propicia la participación real 
de los estudiantes durante todo el proceso de estructuración y desarrollo del proyecto y el desarrollo de la actividad escritora en contextos reales de comunicación.

\section{Referencias bibliográficas}

Arroyo González, R. (2009). Desarrollo metacognitivo y sociocultural de la composición escrita: interculturalidad y tecnologías en la enseñanza de la escritura multilingüe. Granada: Nativola.

Camps, A. (2003). Miradas diversas de la enseñanza y el aprendizaje de la composición escrita. Lectura y vida: revista latinoamericana de lectura.

Cassany, D. (2001). Decálogo de la enseñanza y la composición. Glosas didácticas. Revista on line de la Sociedad Española de Didáctica de la Lengua y la Literatura, 4.

Cassany, D. (1999). Construir la escritura. Barcelona: Paidós.

Cuervo, C., \& Flórez, R. (2000). La escritura como proceso. En Jurado, F. \& Bustamante, G. Los procesos de la escritura. Bogotá: Magisterio.

Díaz Barriga, F. (2003). Cognición situada y estrategias para el aprendizaje significativo. Revista Electrónica de Investigación Educativa, 5(2). Recuperado de http://redie.ens.uabc.mx/ vol5no2/contenido-arceo.html

Elliott, J. (2000). La Investigación-acción en educación. Madrid: Morata.

Fernández Cárdenas, J. (2009). Lengua escrita: habilidades descontextualizadas vs prácticas contextualizadas. En Aprendiendo a escribir juntos: Multimodalidad, conocimiento y discurso. Monterrey: Universidad Autónoma de Nuevo León, pp.50-64.

Ferreiro, E. (1982). Los procesos constructivos de apropiación de la escritura. En E. Ferreiro y M. Gómez Palacio (eds.). Nuevas perspectivas sobre los procesos de lectura y escritura. México: Siglo XXI.

Flórez, R., \& Torrado, M. et al. (2003). Explorando la metacognición: evidencia en actividades de lectura y escritura en niños y niñas de 5 a 10 años de edad. Revista Colombiana de Psicología, (12), pp. 85-98.

Flower, L., \& Hayes, J. (1996). La teoría de la redacción como proceso cognitivo. En Textos en Contexto No 1, los procesos de lectura y escritura. Lectura y vida, (73-107). Recuperado el 20 de marzo de 2015, de http://media.utp.edu.co/referenciasbibliograficas/uploads/ referencias/libro/518-flowers-y-hayes-la-teoriade-la-redaccion-comoprocesocognitivpdfxwyX0-articulo.pdf 
Fons, M. (2004). Leer y escribir para vivir: Alfabetización inicial y uso real de la lengua escrita en la escuela. Barcelona: Graó.

Guardián Fernández, A. (2007). El paradigma cualitativo en la investigación socio-educativa. Costa Rica: IDER.

Jaimes, G. (2001). Teorías del conocimiento e investigación linguística. En Lenguaje y cognición universos humanos. Instituto Caro y Cuervo. Universidad de Salamanca, pp. 109-129.

Jolibert, J., \& Sraiiki, C. (2009). Niños que construyen su poder de leer y escribir. Buenos Aires: Manantial.

Lacón, N., \& Ortega, S. (2008). Cognición, metacognición y escritura. Revista Signos, 41(67), 231-255.

Lerner, D. (2001). Leer y escribir en la escuela: lo real, lo posible y lo necesario. México, DF: Fondo de Cultura Económica.

Martínez, M. (2006). Lainvestigación cualitativa (síntesis conceptual). IPSI Revista de Investigación en Psicología, 9(1), 123-146.

Rincón, G. (2012). Los proyectos de aula y la enseñanza y el aprendizaje del lenguaje escrito. Colombia: Red Colombiana para la Transformación de la Formación Docente en Lenguaje.

Rogoff, B. (1997). Los tres planos de la actividad sociocultural: apropiación participativa, participación guiada y aprendizaje. En J. V. Wertsch, P. Del Rio \& Álvarez, A. (Coord.). La mente sociocultural. Aproximaciones teóricas y aplicadas. (pp.111-128). Madrid: Infancia y Aprendizaje.

SED (2006). "Respuestas grandes para grandes pequeños: Lineamientos Primer Ciclo de Educación Formal en Bogotá, de Preescolar a $2^{\circ}$ grado” Bogotá.

Vasilachis, I. (1997). El pensamiento de Habermas a la luz de una metodología propuesta de acceso a la teoría. Revista Estudios Sociológicos, XV(43). Colegio de México. Recuperado el 13 de julio de 2012, de: codex.colmex.mx:8991/F/?func=service\&doc

Vasilachis, I. (1992). Métodos cualitativos I - Los problemas teórico-metodológicos. Buenos Aires: Centro Editor de América Latina.

Vygotsky, L. S. (1931). La prehistoria del desarrollo del lenguaje escrito. En L. S. Vygotsky, LS. (1995). Obras escogidas (t. III). Madrid: Visor. 